\title{
Simulation tools for scattering corrections in spectrally resolved X-ray Computed Tomography using McXtrace
}

Busi, Matteo; Olsen, Ulrik L.; Knudsen, Erik B.; Frisvad, Jeppe R.; Kehres, Jan; Dreier, Erik S.

Published in:

Optical Engineering

Link to article, DOI:

10.1117/1.OE.57.3.037105

Publication date:

2018

Document Version

Publisher's PDF, also known as Version of record

Link back to DTU Orbit

Citation (APA):

Busi, M., Olsen, U. L., Knudsen, E. B., Frisvad, J. R., Kehres, J., \& Dreier, E. S. (2018). Simulation tools for scattering corrections in spectrally resolved X-ray Computed Tomography using McXtrace. Optical Engineering, 57(3), [037105]. https://doi.org/10.1117/1.OE.57.3.037105

\section{General rights}

Copyright and moral rights for the publications made accessible in the public portal are retained by the authors and/or other copyright owners and it is a condition of accessing publications that users recognise and abide by the legal requirements associated with these rights.

- Users may download and print one copy of any publication from the public portal for the purpose of private study or research.

- You may not further distribute the material or use it for any profit-making activity or commercial gain

- You may freely distribute the URL identifying the publication in the public portal 


\section{Optical Engineering}

\section{Simulation tools for scattering corrections in spectrally resolved $x$ - ray computed tomography using McXtrace}

Matteo Busi

Ulrik L. Olsen

Erik B. Knudsen

Jeppe R. Frisvad

Jan Kehres

Erik S. Dreier

Mohamad Khalil

Kristoffer Haldrup 


\title{
Simulation tools for scattering corrections in spectrally resolved x-ray computed tomography using McXtrace
}

\author{
Matteo Busi, ${ }^{a}$ 网 Ulrik L. Olsen, ${ }^{a}$ Erik B. Knudsen, ${ }^{a}$ Jeppe R. Frisvad, ${ }^{b}$ Jan Kehres, ${ }^{\text {a }}$ Erik S. Dreier, ${ }^{c}$ \\ Mohamad Khalil, ${ }^{a}$ and Kristoffer Haldrup ${ }^{a}$ \\ ${ }^{a}$ NEXMAP Section, DTU Physics, Kongens Lyngby, Denmark

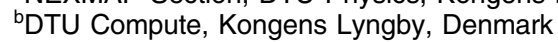 \\ 'Niels Bohr Institute, Copenhagen, Denmark
}

\begin{abstract}
Spectral computed tomography is an emerging imaging method that involves using recently developed energy discriminating photon-counting detectors (PCDs). This technique enables measurements at isolated high-energy ranges, in which the dominating undergoing interaction between the x-ray and the sample is the incoherent scattering. The scattered radiation causes a loss of contrast in the results, and its correction has proven to be a complex problem, due to its dependence on energy, material composition, and geometry. Monte Carlo simulations can utilize a physical model to estimate the scattering contribution to the signal, at the cost of high computational time. We present a fast Monte Carlo simulation tool, based on McXtrace, to predict the energy resolved radiation being scattered and absorbed by objects of complex shapes. We validate the tool through measurements using a CdTe single PCD (Multix ME-100) and use it for scattering correction in a simulation of a spectral CT. We found the correction to account for up to $7 \%$ relative amplification in the reconstructed linear attenuation. It is a useful tool for x-ray CT to obtain a more accurate material discrimination, especially in the high-energy range, where the incoherent scattering interactions become prevailing $(>50 \mathrm{keV})$. (C) 2018 Society of Photo-Optical Instrumentation Engineers (SPIE) [DOI: 19.1117/1].0E.57.3037105]
\end{abstract}

Keywords: computed tomography; spectral computed tomography; multienergy computed tomography; $\mathrm{x}$-ray scattering; Monte Carlo simulations; scattered radiation; fan-beam computed tomography; incoherent scattering.

Paper 171692P received Oct. 25, 2017; accepted for publication Feb. 14, 2018; published online Mar. 14, 2018.

\section{Introduction}

$\mathrm{X}$-ray computed tomography (CT) is an imaging technique that has been developed since the 1970s and is used daily for many purposes, such as medical diagnostics, airport security screening, and food quality $\mathbb{\text { Q }}$ B An x-ray CT acquisition consists in measuring the ratio between the transmitted and incident photons on an object under investigation from multiple angles. To achieve high-quality imaging of the object, it is of key importance to have a good model of the physical interactions between the x-ray photons and the sample. Most of the reconstruction techniques implemented in conventional CT scanners are based on the Bouguer-Beer-Lambert law, where the interactions are assumed to be fully described by an exponential attenuation model. Moreover, the detectors adopted by these instruments are typically based on single or dual energy methods, in which the incoming radiation signal is integrated in the full energy interval, losing the energydependent features. Consequently, in standard commercial $\mathrm{x}$-ray CT scanners, there are two main effects deteriorating the quality of the reconstructions.

First, the x-ray source is typically emission from an anode that generates a polychromatic beam. However, materials absorb the low-energy photons more efficiently than highenergy photons, resulting in cupping and streaking artifacts in the reconstructions due to such beam hardening. Monte Carlo (MC) simulations have been used to predict and correct such effects. ${ }^{\mathbb{\theta}}$ However, it turns out to be challenging in fields, where the sample is complex and the materials have a wide range of attenuating materials. An emerging technique involves the use of single photon-counting detectors (PCDs) ${ }^{\mathbf{1}}$ capable of discriminating the energy of the incoming photons. In this way, under the assumption that the energy resolution is good enough, each measurement can be considered a set of monochromatic acquisitions in what is called spectral CT.

Second, the reconstruction models do not consider that the detected total signal is not only composed of the primary radiation, the photons reaching the detector along a linear path from the source, but also of the scattered radiation, the photons having undergone scattering events within the sample volume. Johns and Yaffe ${ }^{\mathbb{1}}$ have shown that this scattering contribution can produce significant cupping artifacts in fan-beam geometry CT acquisitions. Nevertheless, compared to the cone-beam CT, its impact is substantially decreased due to a reduced volume of the sample being irradiated and the detection solid angle being restricted to the fan-beam plane. For an overview of the recent approaches to correct for this effect, the reader is referred to a review by Rührnschopf and Klingenbeck ${ }^{\mathbb{D}}$ At present, however, most of the solutions for the scattering correction adopted in commercial scanners are based on simple Poisson models of the scattering contribution, which may lead into bias artifacts in the reconstruction.

A more accurate approach is to obtain a direct estimate of the scattered radiation, based on the physical interactions between photons and matter in the CT instrument by an MC simulation of the measurement. A simulation method 
providing spectral scattering estimate in fast time scales has recently been developed and validated, based on a hybrid $\mathrm{MC}$ and deterministic approach. In the latter, the scattering points in the sample are computed by MC simulations, and then deterministic calculations set the particle fractions reaching each detector pixel. Another limitation of current MC simulation methods is that samples are typically rendered as simple polygons or triangle meshes. Therefore, samples of complex shapes and overlapping between each other would dramatically increase computational costs. Last, most of the MC simulation tools proposed at present are based on energy integrating detectors, which are not compatible with spectral CT.

In this work, we present a fully stochastic MC simulation tool for spectral CT x-ray tracing featuring an estimation of the scattered radiation, based on volumetric discretized phantoms, which allows the computation of complex sample geometries without an impact on the time efficiency. Our preliminary investigations of this technique have been presented elsewhere, the pseudocode for our algorithm is therefore not in the main text but deferred to the Appendix. In Sec. 2, we show the methods implemented in the simulation tool and the technique adopted to experimentally detect the scattered radiation contribution. In Sec. B, we validate the simulation tool by comparing the scattering signal obtained by simulation and real acquisitions. Finally, in Sec. \#, we present a simulation study carried out to estimate and correct for the scattered radiation contribution to spectral CT and we draw some conclusions in Sec. 5.

\section{Methods}

In spectral CT, the total detected signal $S_{t}(\vec{r}, E)$ is a function of the pixel position $\vec{r}$ and energy $E$ and can be written as follows:

$$
S_{t}(\vec{r}, E)=S_{p}(\vec{r}, E)+S_{s}(\vec{r}, E)+S_{\mathrm{bkg}}(\vec{r}, E),
$$

where $S_{p}(\vec{r}, E)$ is the primary signal, which is the detected photons not undergoing interactions with the object; $S_{S}(\vec{r}, E)$ is the scattered signal, composed of the photons being scattered by the sample. Last, $S_{\mathrm{bkg}}(\vec{r}, E)$ is the background contribution, composed of the photons being scattered or reflected from the environment and collimating components. In this work, the latter term is not included in the simulation framework as it is highly dependent on the setup of the instrument, and we assume that the system can be optimized to minimize its contribution. Furthermore, in spectral CT, the total signal suffers distortions of the spectral distribution due to detector effects causing the photons to be detected with a certain energy shift. Such effects (e.g., charge sharing, escape peaks, weighting potential, etc.) can be modeled using a unified detector response matrix (DRM), which is applied to each of the real acquisitions on a pixel-by-pixel basis. Effects such as pile-up that depend on the flux of the X-ray beam are not efficiently accounted for by the DRM approach and need to be dealt independently $\square$ Christensen et al. 12 introduced an accurate correction method, adopted in this work, for real acquisitions. This is time efficient and reliable for systems working with a high x-ray flux, up to $5 \mathrm{Mph} / \mathrm{s} / \mathrm{mm}^{2}$.

\subsection{Monte Carlo Simulation Tool}

The simulations framework adopted for this work is McXtrace, $\$$ a software package for MC simulation of X-ray experiments by ray-tracing methods. Rather than tracing individual photons, in this framework, rays are represented by photon entities, and their interactions are simulated by probabilistic weight factors and tracing parameters, such as direction, wave-vector, polarization vector, and phase. The individual parts composing the instrument (e.g., sources, slits, detectors, samples, etc.) are identified as so-called components that can be separately implemented in comparatively few lines of simple code and interact with each sampled ray by applying weight factors operation or altering their parameters. For this work, we have developed a sample component suitable for spectral CT with explicit treatment of the different physical interactions of x-rays incident on objects composed of multiple materials of a given shape. The sample is initialized by loading a volumetric discretized phantom of the object that is made up of a finite number of voxels of a defined size in a 3-D rectangular parallelepiped grid representing the bounding box. In this way, samples composed of objects of complex shape require the same computational cost as simple geometries. Moreover, since the reconstruction images rendered and employed in the forward and back projections of iterative methods are discretized in the same way, the format is well suited for reconstruction algorithms and their parameter optimization. The value in each voxel holds an integer number $i=0,1,2, \ldots, N$, which labels a specific material. For each material, lookup tables are required as input, visualized in Fig. 11.

The first lookup table is composed of the energy parametrized cross-sections for the possible interactions between the X-ray beam and the sample; i.e., photoelectric absorption $\sigma_{\mathrm{ph}}^{i}(E)$, coherent (Rayleigh) $\sigma_{\mathrm{coh}}^{i}(E)$, and incoherent (Compton) $\sigma_{\text {inc }}^{i}(E)$. These values are used to determine the likelihood of each interaction in the component and could, for instance, be loaded from the database administrated by the National Institute of Standards and Technology (NIST) 1 In a simplified approach, assuming that the scattered radiation is deflected out of the detector's range, the total attenuation cross-section can be used ignoring the following component treatment of the scattering. However, as it can be observed in Fig. 1, above 50-keV incoherent scattering interaction is dominant, whereas photoelectric absorption is prevailing for lower energies. Coherent scattering is about 2 orders of magnitude lower than the total attenuation, and only influent in the low energy range.

The remaining two lookup tables are used to sample the coherent and incoherent angular deflection of the ray trajectory when a scattering event occurs and is made up of the coherent and incoherent scattering functions $I_{\text {coh }}^{i}(Q)$ and $I_{\text {inc }}^{i}(Q)$ of each material. The scattering function is treated as the probability distribution function (PDF) of the scattering vector $\vec{Q}$ of amplitude:

$Q=|\vec{Q}|=\frac{4 \pi}{\lambda} \sin \left(\frac{2 \theta}{2}\right)$,

where $\lambda$ is the wavelength of the incident $\mathrm{x}$-ray and $2 \theta$ is the detected scattering angle (i.e., between the incident and the scattered ray). In this way, the scattering vector's amplitude $Q$ can be sampled by the inversion method, $\sqrt{5}$ which involves 

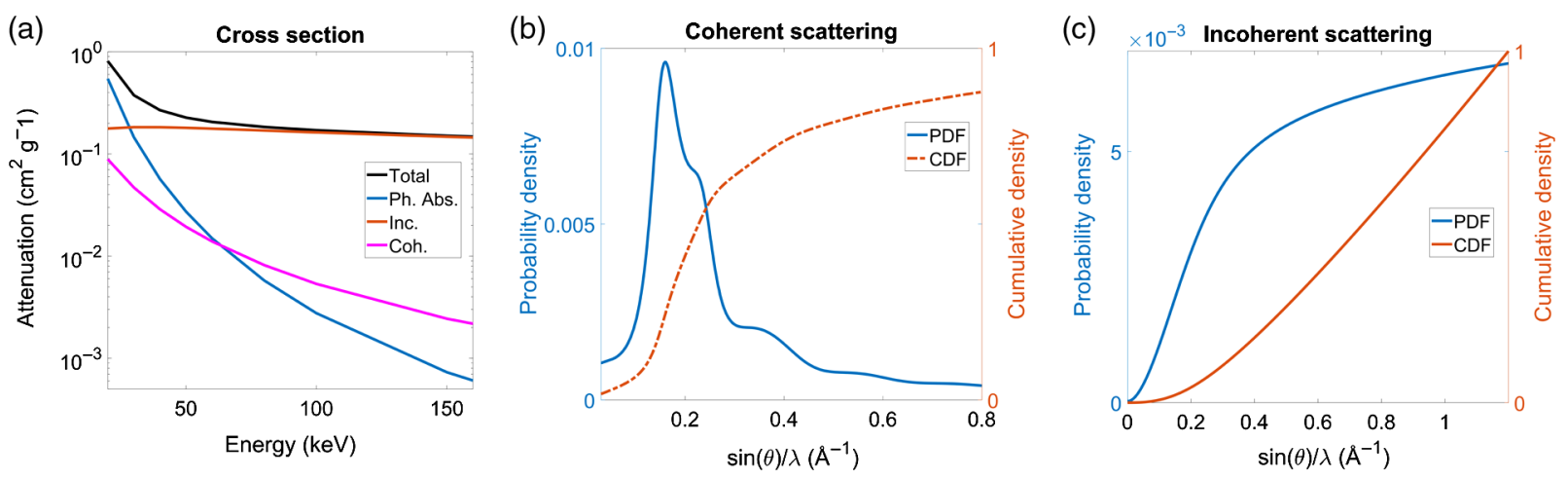

Fig. 1 The lookup tables used by the sample component, exemplified for water. (a) The NIST crosssections are represented for each type of interaction: photoelectric absorption (Ph. Abs.), Rayleigh scattering (Coh.), Compton scattering (Inc.) and total attenuation (total), the sum of the three. Note the logarithmic scale to emphasize each contribution to the total attenuation. (b) and (c) The coherent and incoherent, respectively, scattering functions' probability distribution function (PDF) and their respective cumulative distribution function (CDF) are represented. Note the different scales for the $y$-axis.

computing the cumulative distribution function (CDF) of the distribution and then inverting that function. Since the latter is discrete, the computation of the CDF is simply done by adding up the individual probabilities (normalized to sum 1) for various points of the distribution. The detected scattering angle $2 \theta$ is derived from the sampled $Q$ using Eq. (2) since the energy of the incident ray is a known parameter. The distributions used for $I_{\mathrm{coh}}^{i}(Q)$ in this work have been experimentally determined by the energy dispersive $\mathrm{X}$-ray diffraction method, as described by Kehres et al ${ }^{10}$ and entered into a database of common materials. The stateof-the-art coherent scattering distribution used for water, shown in Fig. 1, is the one presented by Skinner et al. 17 that was made available for download.

To model the distribution of incoherent scattering $I_{\text {inc }}^{i}(Q)$, we have adopted the parametrization presented by Hajdu 18 and Palinkas. 1 As an alternative, the angular deviation of scattering events can be sampled as modeled by the Thomson and Klein-Nishina functions. The azimuthal angle $\phi$ is uniformly sampled in the interval $(0,2 \pi)$, since the radiation emitted by a conventional $x$-ray tube is almost fully unpolarized. Finally, when the scattering event is determined to be of Compton type, the ray's energy parameter is updated to $E_{\text {inc }}$, according to the Compton energy shift relation:21

$$
E_{\mathrm{inc}}=\frac{E}{1+\frac{E}{511 \mathrm{keV}}[1-\cos (\theta)]} .
$$

The projections' runtime of the simulations performed in the upcoming sections is $2 \mathrm{~min}$ on a standard laptop, equipped with i7-6600U quad-core CPUs at $2.60 \mathrm{GHz}$. The speed performance of the scattering estimator is similar and, in some instances, faster than what is reported in some previous recent works for a sample of similar geometrical size ${ }^{8}$ For the simulation of CT experiments, as presented in Sec 4 , several projections can be simulated in parallel in multicore architectures to increase efficiency.

\subsection{Instrumentation}

The instrumentation used for the validation of the scattering estimation of the new scattering component was designed for spectral CT measurements. The X-ray beam was generated by a tungsten anode, and the acceleration voltage and filament current were set to $160 \mathrm{kV}$ and $0.5 \mathrm{~mA}$, respectively. For these operating parameters, the focal spot was $75 \mu \mathrm{m}$. The detector was made up of two 1-D PCD MultixME100 ${ }^{\text {⿴囗十 }}$ modules, composed of $1 \times 128$ pixels of size $0.8 \times$ $0.8 \mathrm{~mm}^{2}$ and each with 128 energy bins of width $1.1 \mathrm{keV}$, evenly distributed between 20 and $160 \mathrm{keV}$. The energy resolution of the detector under high x-ray fluxes is of $6.5 \%$ $(8 \mathrm{keV})$ at $122 \mathrm{keV}$. $\mathrm{A}$ 3-mm thick aluminum filter was placed in front of the source to reduce the beam hardening effects and to suppress the photons with energy below the spectral range of the detector. An energy distribution of the source, shown later in Fig. 5, was obtained by an acquisition of the direct beam (i.e., without the sample being inserted). The characteristic x-ray peaks of the tungsten target are smeared due to the limited detection energy resolution, 203 resulting in a smoother spectrum compared to what is theoretically expected. The source spectrum is used in the simulation as the PDF of the ray's energy parameter. In this way, we simulate a polychromatic beam with the same features as we can measure. The sample used in the work presented here was composed of four glass $\left(\mathrm{SiO}_{2}\right)$ bottles filled with, respectively, water $\left(\mathrm{H}_{2} \mathrm{O}\right)$, hydrogen peroxide $\left(\mathrm{H}_{2} \mathrm{O}_{2}\right)$, powdered sugar $\left(\mathrm{C}_{12} \mathrm{H}_{22} \mathrm{O}_{11}\right)$, a powdered PETN explosive simulant, and an aluminum rod. The sample, sketched in Fig. 2, was designed to highlight the challenge of automated accurate threat detection in security screening, as they are innocuous and harmful materials, which have very close values of efficient atomic number $\left(Z_{\text {eff }}\right)$ and density relative to water $\left(\rho_{\text {rel }}\right)$.

\section{Experimental Validation}

The method adapted to experimentally measure the detected radiation being scattered from the sample $S_{s}(\vec{r}, E)$ was the collimator shadow. The latter was shown to be an accurate empirical technique ${ }^{24}$ and suits our beam collimation choice of a fan-beam. This technique, sketched in Fig. 2, combines a fan-beam collimation with a 2-D flat detector, to obtain considerably extended surfaces in which only the scattered radiation is present. The collimation was obtained by a vertical slit composed of two tungsten blades. The 2-D flat detector was reproduced by sequentially translating in 


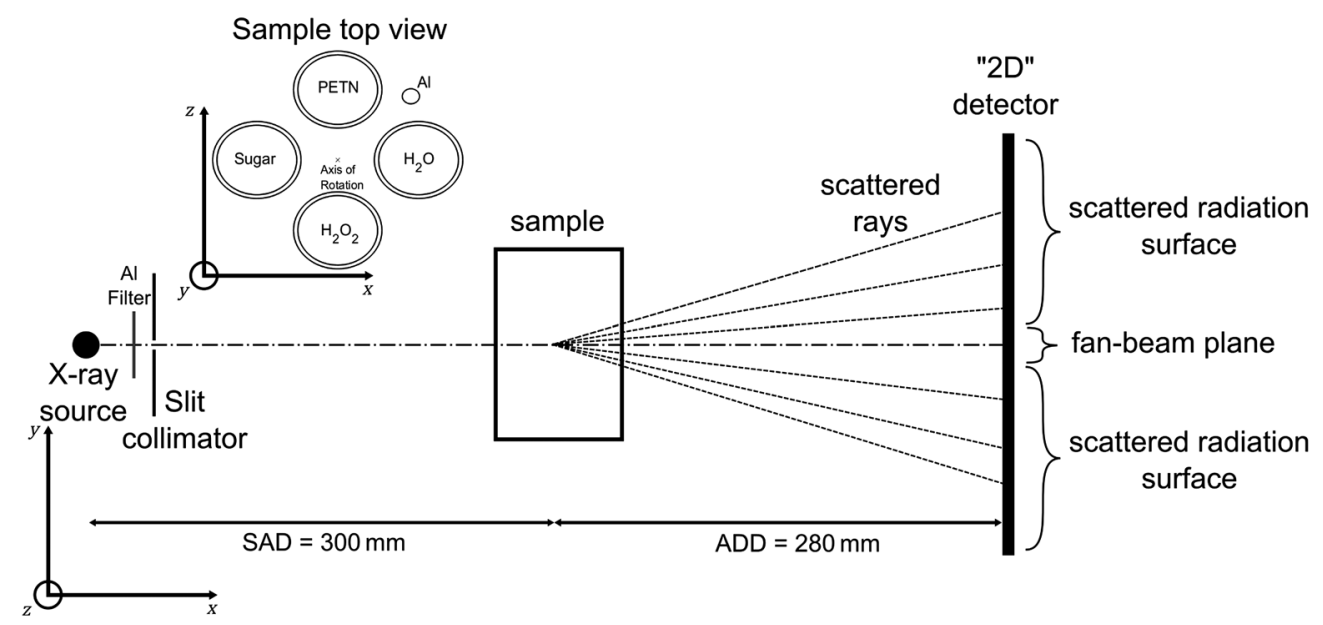

Fig. 2 Collimator shadow method: the $x$-ray beam is collimated into a fan-beam geometry. The sample is placed at a source-to-axis distance $(S A D)=300 \mathrm{~mm}$ from the detector, whereas the 1-D detector, placed at an axis-to-detector distance $(A D D)=280 \mathrm{~mm}$ from the sample, was composed of 256 pixels in the $z$-direction and is vertically translated in the $y$-direction to reproduce a 2-D detector. In the detector area shadowed by the collimator, the signal is only composed of the scattered radiation.

70 steps vertically our linear detector, resulting in an array of $70 \times 256$ pixels 2 -D detector. We restricted the detection energy range to the interval between 40 and $160 \mathrm{keV}$ to disregard energy channels with low photon statistics, as they would display accentuated distortions in the spectral shape due to insufficient signal to noise ratio.

In this technique, in the shadows cast by the collimator (i.e., outside of the fan-beam plane), the primary signal is absent, $S_{p}(\vec{r}, E)=0$. Assuming absence of air scattering, the isolation of the sample scattering contribution $S_{S}(\vec{r}, E)$ can therefore be obtained by the subtraction between the acquisition with and without the sample, $S(\vec{r}, E)$ and $S_{0}(\vec{r}, E)$, respectively, to reduce the environmental noise:

$$
\begin{aligned}
S_{s}(\vec{r}, E) & =S(\vec{r}, E)-S_{0}(\vec{r}, E) \\
& =S_{s}(\vec{r}, E)+S_{\mathrm{bkg}}(\vec{r}, E)-S_{\mathrm{bkg}}(\vec{r}, E) .
\end{aligned}
$$

A mathematical phantom of the sample of $150 \times 150$ vertically homogeneous voxels of size $0.667 \mathrm{~mm}$ was generated and loaded into the simulation component described in Sec. 2, to estimate the total signal $\tilde{S}(\vec{r}, E)$ and its respective sample scattering contribution $\tilde{S}_{s}(\vec{r}, E)$. The geometrical setup of the instrumentation in the simulations was set to match the experimental setup.

\subsection{Simulation Output}

The runtime for simulating each projection, sampling $10^{6}$ rays, was $2 \mathrm{~min}$. To reduce further the computational efforts of the MC simulations, we applied a 3-D Gaussian blurring to the spectral scattering projections $\tilde{S}_{s}(\vec{r}, E)$, inspired by the accelerated simulation method presented by Colijn and Beekman. The method consists in filtering the projections with a 2-D Gaussian smoothing kernel, with standard deviation $\sigma$ being the blurring width. We introduce a tradeoff between noise and blur (variance and bias) in the simulation output using fixed bandwidth kernel density estimation with a Gaussian kernel ${ }^{\mathbb{6}}$ We use a 3-D Gaussian kernel and vectorial form of the blurring width $\vec{\sigma}=\left(\sigma_{z}, \sigma_{y}, \sigma_{E}\right)$, composed of the individual blurring widths in the $z$ - and $y$-axes and the energy domain. The optimal choice of $\vec{\sigma}$ depends on the number of rays being sampled and the geometrical parameters of the system. For this work, the blurring width has been found by the minimization of the combined root mean squared error, $\operatorname{RMSE}\left(\sigma_{z}, \sigma_{y}, \sigma_{E}\right)$, with a reference considered as true image:

$$
\begin{aligned}
& \operatorname{RMSE}\left(\sigma_{z}, \sigma_{y}, \sigma_{E}\right) \\
& =\sqrt{\sum_{i=1}^{N_{z}} \sum_{j=1}^{N_{y}} \sum_{k=1}^{N_{E}} \frac{\left(\tilde{S}_{s}\left(z_{i}, y_{j}, E_{k}\right)-\tilde{S}_{\mathrm{ref}}\left(z_{i}, y_{j}, E_{k}\right)\right)^{2}}{N_{z} N_{y} N_{E}}},
\end{aligned}
$$

therefore, $\tilde{S}_{\text {ref }}(\vec{r}, E)$ is the scattering projection used as true reference, obtained by simulating $10^{9}$ rays; $N_{z}$ and $N_{y}$ are the numbers of pixels in the $z$ - and $y$-direction, respectively; and $N_{E}$ is the number of energy bins of the detector. Figure 3 shows $\tilde{S}_{s}\left(z_{i}, y_{j}, E_{k}\right), \tilde{S}_{\text {ref }}\left(z_{i}, y_{j}, E_{k}\right)$, and the filtering result, for blurring width $\vec{\sigma}=(9,4.14,1.71)$. For a better visualization of the results, the scattering projections shown in the following figures have been energy integrated into $\tilde{S}_{s}(z, y)=$ $\sum_{k=1}^{N_{E}} \tilde{S}_{s}\left(\vec{r}, E_{k}\right)$ and spatially integrated into $\tilde{S}_{s}(E)=$ $\sum_{i=1}^{N_{x}} \sum_{j=1}^{N_{y}} \tilde{S}_{s}\left(z_{i}, y_{j}, E\right)$. The drop in intensity that can be observed within the fan-beam plane indicates that the photons being scattered to the fan-beam plane are less likely than the one being scattered out of it. This is the reason underlying the apparition of the two lobes of high intensity in the vicinity that would not be obtained by rather having a cone-beam collimation.

Figure 7 shows the output of the simulation, displaying the individual types of scattering events contributing to the total scattering signal, and their respective spectral and spatial distribution. It can be observed that both the incoherent and multiple scattering (i.e., when the photons are scattered more than once in the same path tracing) are heavily spread in a broad range of angles centered in the proximity of the sample, indicating that information about the geometrical structure of the sample is completely lost. A comparison between the coherent and incoherent scattering instead 
(a)
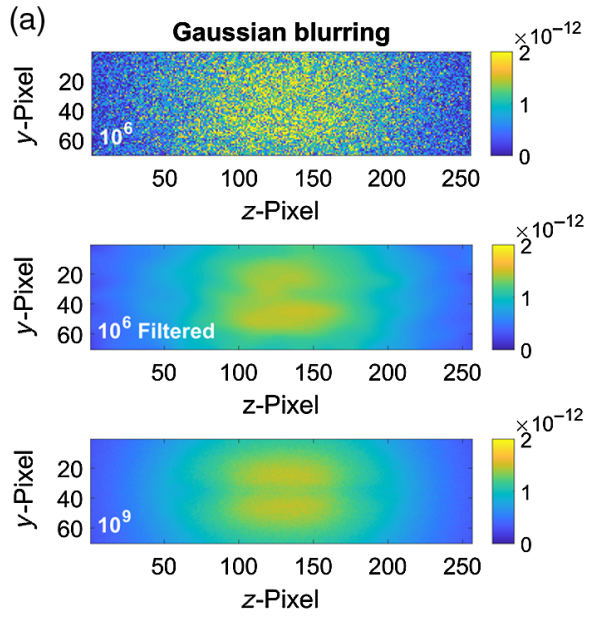

(b)

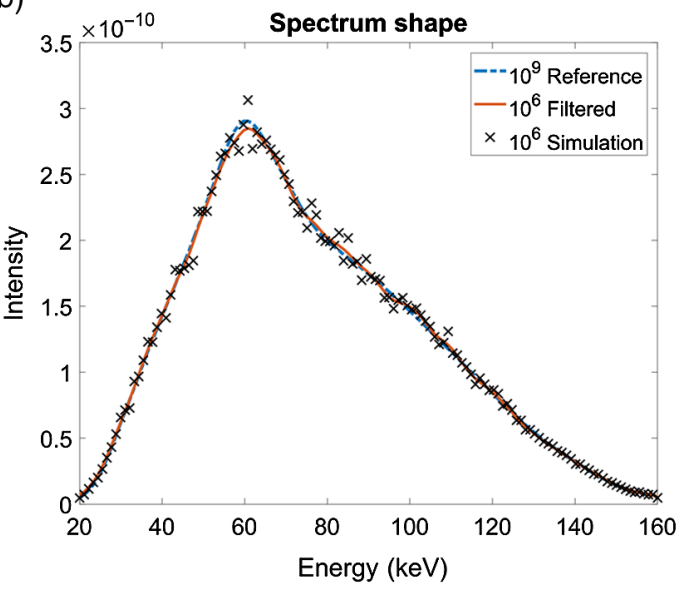

Fig. 3 Visualization of the effect of the 3-D Gaussian blurring. (a) Starting from the top frame toward the bottom are shown the energy integrated scattering estimate $\tilde{S}_{s}(\vec{r})$, the result of the filtering and the reference $\tilde{S}_{\text {ref }}(r)$. (b) The spectral distribution of the respective spatial integrated scattering projections $\tilde{S}_{s}(\vec{E})$.
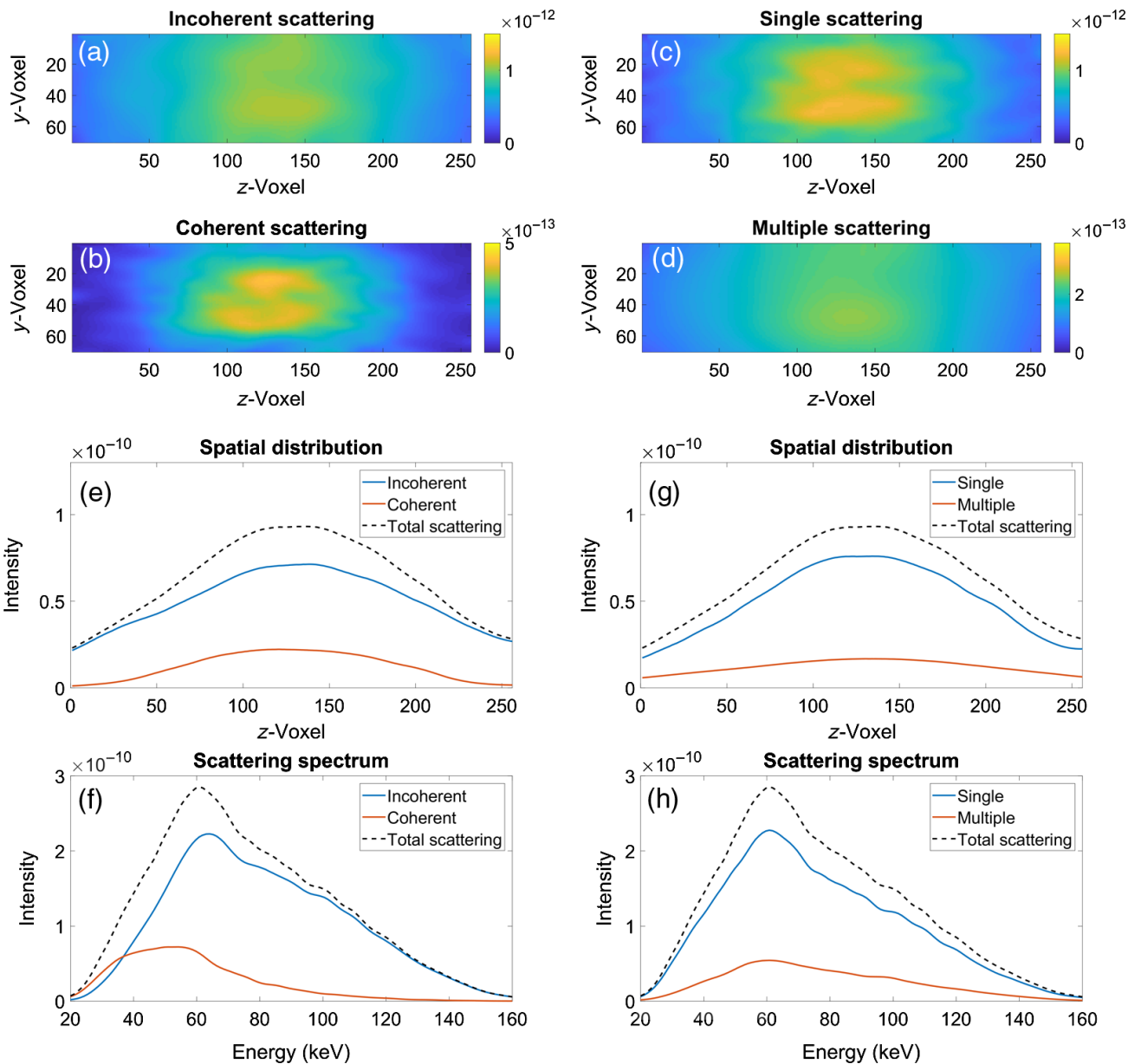

Fig. 4 Simulation output of the scattering contributions to the total signal $S_{t}(\vec{r}, E)$. (a)-(d) Energy integrated incoherent, coherent, single and multiple scattering profiles. (e)-(h) Spatial integrated and energy integrated distribution of the individual $x$-ray scattering profiles. The dashed black line is the total scattering signal, whereas the blue and red lines are its incoherent and coherent contributions in $(e, f)$, and its single and multiple contributions in $(g, h)$. Note that the intensity scale of $(b)$ is three times lower than (a), whereas the intensity scale of panel (d) is four times lower than panel (c). 

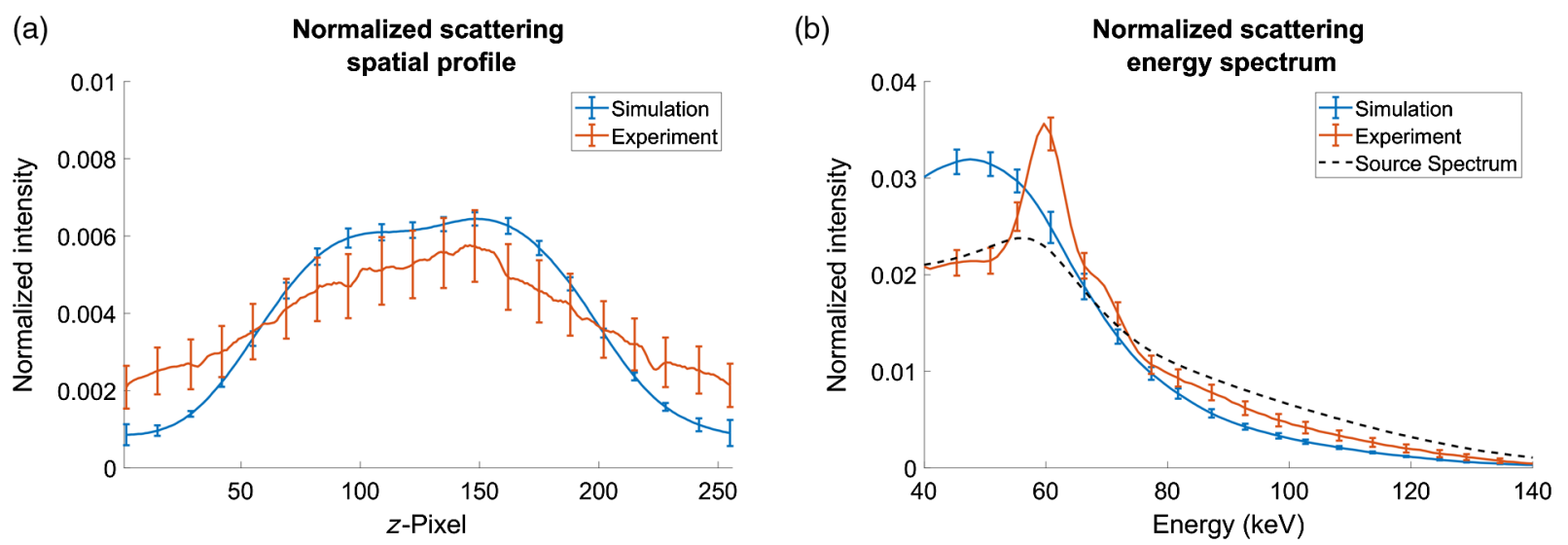

Fig. 5 Validation of the simulation tool. (a) The normalized estimated (simulation) and measured (experiment) scattering signal, integrated over the energy and the $y$-axis area shadowed by the collimator are plotted. (b) Their respective spectral distribution and the source spectrum (source spectrum) are plotted. The error bars are shown every 20th point.

suggests that the former contributes largely to the low-energy part of the energy spectrum and, despite that, is heavily centered toward the small angles compared to the latter. It is also evident that above $50 \mathrm{keV}$, the scattering profile is dominated by the incoherent scattering events. A look at the multiple scattering profiles suggests that it can be treated as a constant offset relative to the single scattering, if the purpose is to employ fast and simplified computational models. Last, it should be noted that the simulation output is on an arbitrary scale since it is a probability map. Therefore, the data should be interpreted after it has been properly rescaled according to the beam flux, which could be, for example, determined by placing a virtual monitor before the sample. However, for most applications, this procedure is not necessary as it is the case of the reconstruction techniques, in which the datasets are normalized in the preprocessing of the attenuation $A(\vec{r}, E)$ according to Eq. (円):

$A(\vec{r}, E)=-\log \frac{S(\vec{r}, E)}{S_{0}(\vec{r}, E)}$.

\subsection{Comparisons}

Figure 5 displays the comparisons of the scattered radiation obtained by both the simulation and experiment, and their respective spatial and energy profiles. The spatial profile of the two signals shows a qualitative good match, which does not completely hold for the energy distribution. A visual comparison of the experimental and simulated spectral distributions indicates an overestimation of the high-energy and, thus, supposedly the incoherent scattering events. Concurrently, the experimental spectral distribution displays sharper peaks centered around the characteristic peaks of tungsten. This indicates a relatively large environmental noise in addition to the scattering signal, which can be caused, for example, by the scattering of the slits used for the front collimation of the beam since they are made of tungsten. That can be due to direct beam reflection or scattering from other parts composing the instrument. Therefore, we attribute the reason for the discrepancy to the experimental complexity in achieving the ideal collimation of a fanbeam and in reducing the background radiation incoming from the system environment. A more accurate detection of the spectral distribution of the scattered radiation could be achieved by a better shielding of the background radiation, which was beyond the purpose of this work.

Another limitation of the simulation tool is the dependence on the quality of the models used for the coherent scattering angular deviation sampling. For certain samples, for instance, the noise removal can be challenging due to the relatively low count rate of the technique, leading to imprecisions in the measured distribution. Furthermore, the instrumental constraints prevent the determination of the coherent scattering functions $I_{\mathrm{coh}}^{i}(Q)$ in the low- $Q$ limit, due to the presence of the direct beam. Last, the analytical approximations used for the incoherent scattering functions are required to satisfy the validity range $\sin (\theta) / \lambda<1.2 \sqrt{19}$ posing a limit to the high angles.

\section{Computed Tomography Scattering Correction}

In the last part of the work, we have simulated a spectral CT experiment of 101 projections evenly distributed between $\omega=(0,2 \pi)$. For each projection, $10^{6}$ rays are traced followed by the Gaussian blurring of the scattering projections. The specifications of the instrumentation and of the sample are sketched in Fig. 2 and described in Sec. \&. The reconstruction method adopted was simultaneous iterative reconstruction technique (SIRT), implemented in the ASTRA reconstruction toolbox. 27 The latter maps the attenuation simulated projections into volumetric representation of the linear attenuation of the sample $\mu(\vec{r}, E)$. To assess the impact of the scattered radiation, we have performed slice reconstructions $\mu_{t}(\vec{r}, E)$ and $\mu_{p}(\vec{r}, E)$ of, respectively, the total attenuation projections $A_{t}(\vec{r}, E)$ and of the scattering corrected primary attenuation projections $A_{p}(\vec{r}, E)$. These are obtained by inserting the simulated total signal $\tilde{S}_{t}(\vec{r}, E)$ and primary radiation $\tilde{S}_{p}(\vec{r}, E)=\tilde{S}_{t}(\vec{r}, E)-\widetilde{S}_{s}(\vec{r}, E)$, respectively, in Eq. (円). It must be noted that following Eqs. (11) and (\#), since the total signal is corrupted by the scattered radiation, the total attenuation yields a lower value compared to the true attenuation.

The reconstructions shown in Fig. 6 were carried out by first merging the energy channels between 92.7 and $160 \mathrm{keV}$. That is a considerably high-energy interval, such as the 

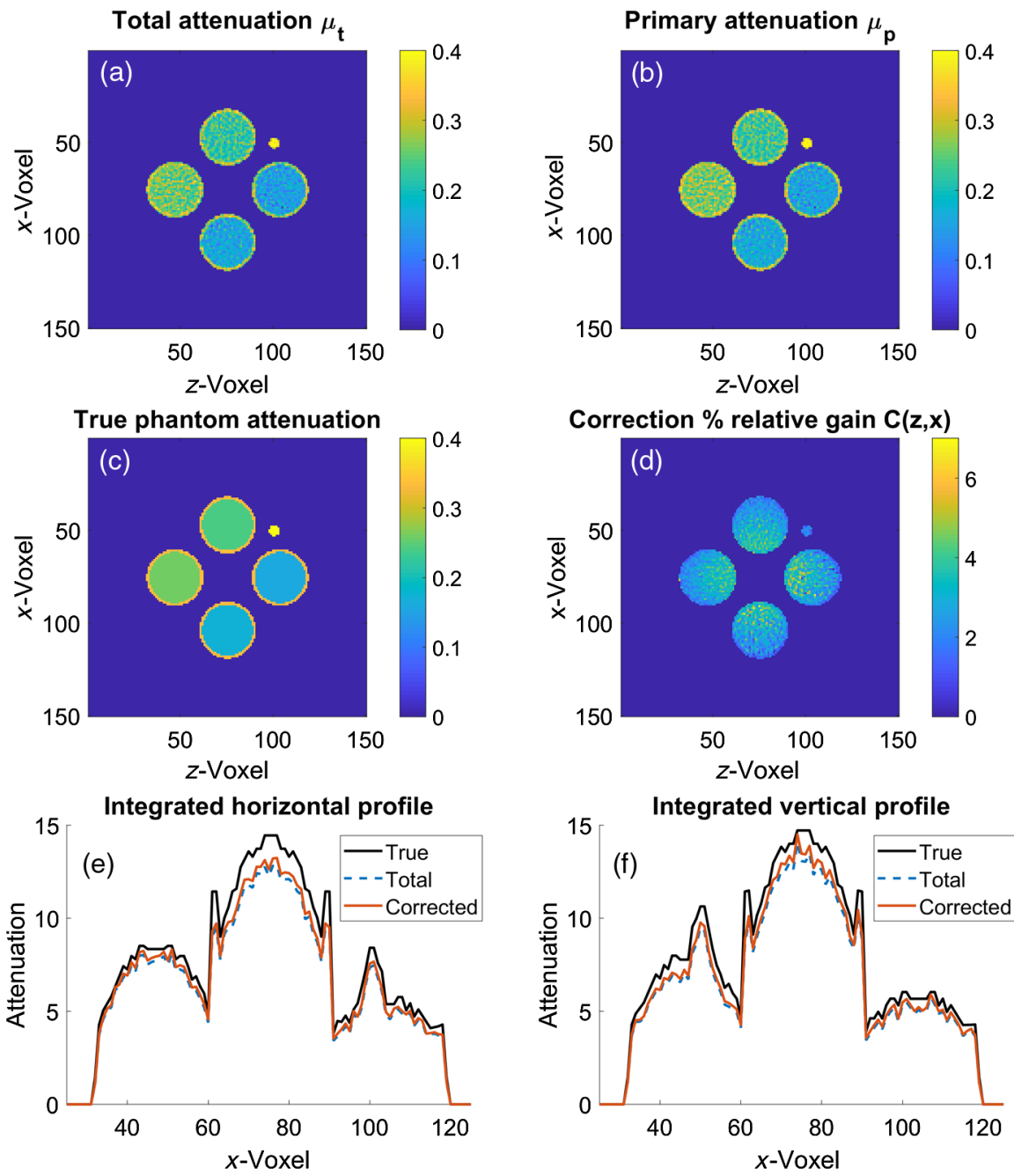

Fig. $6(a, b)$ SIRT reconstruction of the total and scattering corrected primary attenuation. (c) Sample phantom used in the simulations. The color scale in frames $(a-c)$ represents the linear attenuation $\mu$ value held by the voxel. (d) Percentile amplification of the reconstruction value using the corrected attenuation. (e,f) Spatially integrated horizontal and vertical profiles of the images, obtained by summing the values of each row and column, respectively.

ones often implemented in dual-energy $\mathrm{CT}$ techniques for nondestructive testing and security applications, especially for the retrieval of a material's electron density $\rho_{\mathrm{e}} \cdot \mathrm{Q}^{\mathrm{S}}$ The simulation method holds valid for lower energies, even though the correction impact becomes less significant due to a reduced number of scattering events. In Fig. 6, the attenuation profiles have been spatially integrated in a similar way as the scattering projections for a better display of the results. Since the corrected attenuation $\mu_{p}(\vec{r}, E)$ should always be greater than the uncorrected $\mu_{t}(\vec{r}, E)$, we have introduced a correction percentile relative gain $C(\vec{r}, E)$ :

$C(\vec{r}, E)=100 \% \frac{\mu_{p}(\vec{r}, E)-\mu_{t}(\vec{r}, E)}{\mu_{t}(\vec{r}, E)}$,

to quantify the relative change of the scattering correction in the reconstruction of the linear attenuation value $\mu(E)$.

\subsection{Discussion of Results}

It was found that for this energy interval, the correction $C(x, y)$ accounts for an amplification in $\mu(E)$ that can be up to $7 \%$ and increases as the center of mass of the entire sample is approached. It was also observed that it becomes slightly higher for liquid materials, due to their lower linear attenuation, as compared to other materials composing our sample. This indicates that treating the scattering contribution as a mere scaling factor applied to the total attenuation may lead to artifacts, especially in the high-energy range, where the scattering influence becomes increasingly relevant. It is also evident that, by comparing the aluminum and the glass content values, the scattering contribution is highly dependent on the volume covered by each object. Despite the fact that the larger objects of the sample are subject to more significant loss of contrast, the reconstructions do not exhibit the otherwise commonly occurring cupping artifacts. The reason for this is the relatively limited size of the sample and the collimation of the fan-beam.

To evaluate the impact of the scattering correction in the energy domain, we have performed a slice reconstruction of each individual energy channel. The surface covered by each material $i$ was segmented and used to retrieve energy resolved mean linear attenuation $\mu^{i}(E)$ reconstructed by 
(a)

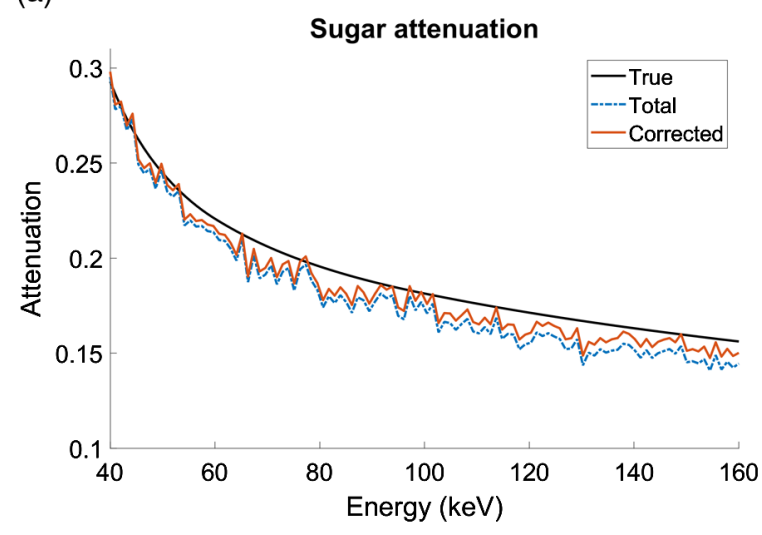

(b)

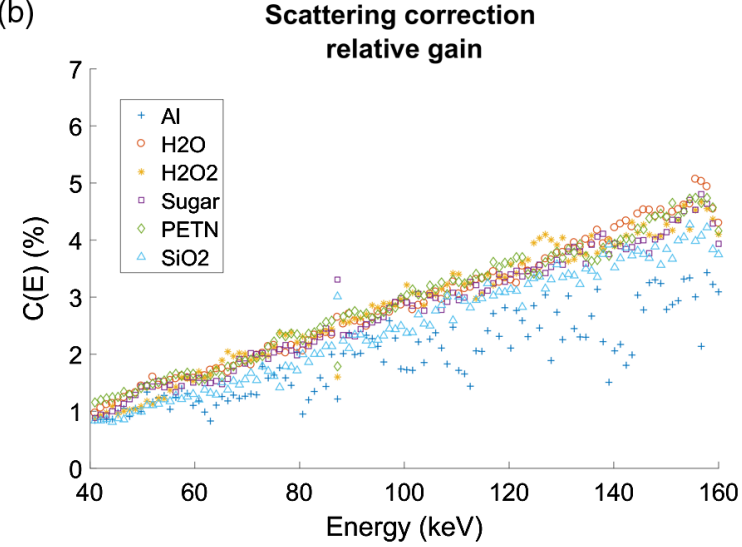

Fig. 7 (a) Mean value of the energy resolved reconstructed attenuation value in the sugar surface, using the total attenuation (total), the primary attenuation (corrected), and the theoretical expected attenuation (true). (b) Spectral profiles of the correction relative gain for each material.

using the total and scattering corrected primary attenuation. Both energy distributions are shown in Fig. 7 for powdered sugar and compared to its respective tabulated values, used in the look-up tables. It was observed that the correction aids in reconstruction of the linear attenuation $\mu$, as it is brought closer to the tabulated linear attenuation. It has to be noted, however, that the scattering signal subtraction correction method used here is merely a preliminary procedure. A more refined approach would include the scattering estimator in the forward model of iterative reconstruction techniques. In this way, the illposedness of the reconstruction problem would be reduced and the gap observed in Fig. 7 , which is due to counting statistics and missing data, will be further closed leading to a more robust solution.

The energy distribution of the correction relative gain $C(E)$ was then derived for each material and is shown in Fig. ㄱ. A comparison between the correction relative gain $C(E)$ for aluminum and the glass contents suggests that larger volumes are more subject to scatter artifacts. Furthermore, the latter increases with the energy reaching values up to $6 \%$, indicating that CT scanners operating in high-energy regimes require scattering correction algorithms for an adequate reconstruction of the linear attenuation. On the other hand, CT reconstruction of low-energy projections with fan-beam collimation seems to be only slightly influenced by the scattered radiation, regardless of the volume and composition of the material.

\section{Conclusions}

We have presented a fully stochastic simulation tool for $\mathrm{x}$-ray spectral CT, featuring a computationally fast estimate of the scattered radiation, which is particularly compatible with objects of complex shape. The tool is open access and runs in the well-established McXtrace software package..$^{-1}$ It has been validated by an experiment designed to detect the radiation being scattered by the sample only. We have discussed the current limitations of the simulation tool and possible strategies to overcome them. In a preliminary approach, we have shown how the scattering estimate can improve the attenuation reconstruction by a restoration of the primary radiation $\tilde{S}_{p}(\vec{r}, E)$, performed as a simple subtraction of the estimated scattering contribution $S_{S}(\vec{r}, E)$. It was found that especially at high energies, where the incoherent scattering events are dominant, the correction is useful to reconstruct a more accurate linear attenuation value and, therefore, improves the contrast between different materials. This is expected to aid the automated segmentation procedures leading to an advance in the material

Algorithm 1 Pseudocode of the McXtrace object component.

Data: Object's phantom and material densities $\rho^{i}$, cross sections $\sigma^{i}(E)$ and scattering functions $I_{\text {coh }}^{i}(Q)$ and $l_{\text {inc }}^{i}(Q)$

Result: Energy resolved total and scattering signal from the interaction between $\mathrm{x}$-rays and the object

initialize system's geometry and variables;

set the step length $s_{l}$;

while ray is within the object do

if ray is scattered then

if Compton scattering then

sample scattering angle $\theta$ from $l_{\text {inc }}^{i}(Q)$;

update ray direction according to $\theta$;

update to Compton energy with Eq. (3);

else

sample scattering angle $\theta$ from $l_{\operatorname{coh}}^{i}(Q)$;

update ray direction according to $\theta$;

end

end

read photoelectric absorption attenuation $\mu(E)=\rho^{i} \sigma_{\mathrm{ph}}^{i}(E)$;

ray travels the step length $s_{\text {; }}$;

increment the cumulative photoelectric absorption attenuation along the trace $\mu(E) I=\mu(E) I+\mu(E) s_{l}$;

end

apply final photoelectric absorption attenuation to the ray: $e^{-\mu(E) \prime}$; 
classification. In future work, we aim to incorporate the scattering estimation in the forward model of a model-based iterative reconstruction technique, to improve even further the efficiency of the algorithm. This could be done, for instance, by, after a certain iteration number of the $\mathrm{CT}$ reconstruction, retrieving a preliminary volumetric representation of the object. The latter would be then used by the MC simulation tool to obtain the direct estimate of the scattering contribution.

\section{Appendix: Sample Component Pseudocode}

The pseudocode of the sample component developed for McXtrace is shown in Algorithm 11. Therefore, the step length $s_{l}$ represents the rate at which the interaction probabilities are checked while the ray is traced through the sample up to the final length $l$. In our simulations, $s_{l}$ was set to a value three times smaller than the voxel size. At the first step of each ray traced, the step length was additionally multiplied by a random generated number to reduce artifacts induced by the tracing regularity ${ }^{29} \mu(E)$ represent the photoelectric absorption linear attenuation, whereas $\rho^{i}$ and $\sigma_{\mathrm{ph}}^{i}(E)$ are, respectively, the density relative to water and the photoelectric absorption cross-section of each material.

In this method, the photoelectric absorption interaction is taken into account by incrementing at each step the outgoing photoelectric linear absorption. The scattering interaction type is determined by generating a random number $\xi=U(0,1)$, with $U(-n, n)$ being the uniform distribution between $-n$ and $n$, and comparing it with the tabulated scattering cross-sections:

Scattering type $= \begin{cases}\text { Compton, } & \text { if } \xi<\left[1-e^{-\rho \cdot \mathrm{s}_{1} \cdot \sigma_{\mathrm{inc}}^{i}(E)}\right] \\ \text { Rayleigh, } & \text { if }\left(1-e^{-\rho \cdot \mathrm{s}_{1} \cdot \sigma_{\mathrm{inc}}^{i}(E)}\right)<\xi<\left[1-e^{-\rho \cdot \mathrm{s}_{1} \cdot\left(\sigma_{\mathrm{coh}}^{i}(E)+\sigma_{\mathrm{inc}}^{i}(E)\right)}\right]\end{cases}$

\section{Acknowledgments}

The authors would like to acknowledge Innovation Fund Denmark for financing their respective work. The authors have no relevant financial interests in the manuscript and no other potential conflicts of interest.

\section{References}

1. W. A. Kalender, "X-ray computed tomography," Phys. Med. Biol. 51, R29-R43 (2006).

2. K. Wells and D. A. Bradley, "A review of x-ray explosives detection techniques for checked baggage," Appl. Radiat. Isot. 70, 1729-1746 (2012).

3. H. Kraggerud et al., "X-ray images for the control of eye formation in cheese," "nt. J. Dairy Technol. 62, 147-153 (2009).

4. M. Thomsen et al., "Prediction of beam hardening artefacts in computed tomography using Monte Carlo simulations," Nucl. Instrum. Methods Phys. Res., Sect. B 342, 314-320 (2015).

5. A. Gorecki et al., "Comparing performances of a CdTe x-ray spectroscopic detector and an $\mathrm{x}$-ray dual-energy sandwich detector," . Instrum. 8(11), P11011 (2013)

6. P. C. Johns and M. Yaffe, "Scattered radiation in fan beam imaging systems," Med. Phys. 9(2), 231-239 (1982).

7. E.-P. Rührnschopf and K. Klingenbeck, "A general framework and review of scatter correction methods in X-ray cone-beam computerized tomography. Part 1: scatter compensation approaches," Hed. Phys. 38(7), 4296-4311 (2011).

8. A. Sossin et al., "Fast scattering simulation tool for multi-energy x-ray imaging," Nucl. Instrum. Methods Phys. Res., Sect. A 802, 60-66 (2015).

9. M. Busi et al., "A Monte Carlo simulation of scattering reduction in spectral x-ray computed tomography," Proc. SPIE 10388, $103880 \mathrm{P}$ (2017).

10. G. Bootsma, F. Verhaegen, and D. Jaffray, "Spatial frequency spectrum of the x-ray scatter distribution in CBCT projections," Med. Phys. 40(11), 111901 (2013).

11. J. Cammin et al., "A cascaded model of spectral distortions due to spectral response effects and pulse pileup effects in a photon-counting x-ray detector for CT," Med. Phys. 41(4), 041905 (2014).

12. E. D. Christensen et al.," "Spectral correction algorithm for multispectral CdTe x-ray detectors," Proc. SPIA 10393, 103930H (2017).

13. E. Bergbäck Knudsen et al., "McXtrace: a Monte Carlo software package for simulating x-ray optics, beamlines and experiments," t. Appl. Crystallogr. 46(3), 679-696 (2013).

14. M. J. Berger and J. H. Hubbell, "XCOM: photon cross sections on a personal computer," No. NBSIR-87-3597, Center for Radiation Research, National Bureau of Standards, Washington, DC (1987).

15. R. Y. Rubinstein and D. P. Kroese, Simulation and the Monte Carlo Method, Vol. 10, John Wiley \& Sons (2016).

16. J. Kehres, M. Lyksborg, and U. L. Olsen, "Threat detection of liquid explosives and precursors from their x-ray scattering pattern using energy dispersive detector technology," Proc. SPIE 10393, 1039302
(2017).
17. L. B. Skinner et al., "Benchmark oxygen-oxygen pair-distribution function of ambient water from x-ray diffraction measurements with a wide Q-range," T. Chem. Phys. 138, 074506 (2013).

18. F. Hajdu, "Revised parameters of the analytic fits for coherent and incoherent scattered x-ray intensities of the first 36 atoms," Acta Cryst. A 28(3), 250-252 (1972).

19. G. Palinkas, "Analytic approximations for the incoherent x-ray intensities of the atoms from Ca to Am," Acta Cryst. A 29(1), 10-12 (1973).

20. J. Baró et al., "Analytical cross sections for Monte Carlo simulation of photon transport," Radiat. Phys. Chem. 44(5), 531-552 (1994).

21. A. H. Compton, "A quantum theory of the scattering of x-rays by light elements," Phys. Rev. 21(5), 483-502 (1923).

22. A. Brambilla et al., "Fast CdTe and CdZnTe semiconductor detector arrays for spectroscopic x-ray imaging," IEEE Trans. Nucl. Sci. 60(1), 408-415 (2013).

23. A. A. Dooraghi et al., "Characterization of a spectroscopic detector for application in x-ray computed tomography," Proc. SPIE 10391, $103911 \mathrm{G}$ (2017).

24. A. Akbarzadeh et al., "Measurement of scattered radiation in a volumetric 64-slice CT scanner using three experimental techniques," Phys. Med. Biol. 55, 2269-2280 (2010).

25. A. P. Colijn and F. J. Beekman, "Accelerated simulation of cone beam Xray scatter projections," IEEE Trans. Med. Imaging 23, 584-590 (2004).

26. B. W. Silverman, Density Estimation for Statistics and Data Analysis, Vol. 26, CRC Press (1986).

27. W. van Aarle et al., "The ASTRA toolbox: a platform for advanced algorithm development in electron tomography," Ultramicroscopy 157, 35-47 (2015).

28. S. G. Azevedo et al., "System-independent characterization of materials using dual-energy computed tomography," EEEE Trans. Nucl. Sci. 63(1), 341-350 (2016).

29. M. Pauly, T. Kollig, and A. Keller, "Metropolis light transport for participating media," in Proc. of EGWR, Rendering Techniques, pp. 11-391, Springer (2000).

Matteo Busi is a PhD student at Technical University of Denmark (Kgs. Lyngby, Denmark). He received his BS degree in physics from the Universitá degli Studi di Parma (Parma, Italy) and his MS degree in mathematics and physics from the Universitetet i Stavanger (Stavanger, Norway) in 2014 and 2016, respectively. He is a member of SPIE.

Ulrik L. Olsen is a senior research engineer at DTU physics since 2013. Funded by Innovation Fund Denmark to develop applications using high flux multispectral x-ray detection technology and currently project leader on CIL2018, a multidisciplinary effort made to reduce the human operator involvement by $50 \%$ for checked-in luggage. $\mathrm{He}$ was previously employed at National Laboratory RIS $\varnothing$, Denmark, as $\mathrm{PhD}$ (2005) and later postdoc working with developments of x-ray sensors.

Erik B. Knudsen is a research engineer at DTU Physics (Kgs. Lyngby, Denmark). He received his PhD degree in photonic crystal 
fiber optics. His main research interests are $x$-ray and neutron scattering computer simulations with emphasis on coherent $\mathrm{x}$-rays and polarized neutrons, as well as $\mathrm{x}$-ray telescope simulations. He is a core developer of McXtrace and McStas simulation packages (since 2007).

Jeppe R. Frisvad is an associate professor at Technical University of Denmark (DTU). He received his MSc (Eng) degree in applied mathematics (2004) and PhD degree in computer graphics (2008) from DTU. His research interests are mainly material appearance modeling, realistic rendering, and light scattering. As a highlight, his work includes development of the first diffusion dipole for subsurface scattering based on ray sources.

Jan Kehres is a research engineer at DTU physics (Kongens Lyngby, Denmark). He received his PhD for in operando investigation of catalyst nanoparticles using $\mathrm{x}$-ray scattering and continued in this field of research as a postdoctoral researcher. His current field of research is the application of energy dispersive detectors for $\mathrm{x}$-ray scattering and advanced imaging modalities with a focus on material identification of illicit materials for security applications.
Erik S. Dreier is a PhD student at the Niels Bohr Institute, University of Copenhagen (Denmark). He received both his BS (2013) and MS (2016) degrees in physics from the University of Copenhagen. His research is focused on finding possible new applications and characterization of novel single photon counting x-ray detectors. $\mathrm{He}$ is a member of SPIE.

Mohamad Khalil is a postdoctoral researcher at Technical University of Denmark (Kgs. Lyngby, Denmark). He received his MS degree in high-energy physics from University Paris Pierre et Marie Curie (Paris, France) in 2011 and his PhD degree from University Paris Denis Diderot (Paris, France) and University of Ferrara (Ferrara, Italy) in December 2014.

Kristoffer Haldrup obtained his $\mathrm{PhD}$ degree from Niels Bohr Institute in 2007, working at Risø National Laboratory in Denmark. He then worked as a postdoc at the Centre for Molecular Movies located at the University of Copenhagen. In 2011 to 2012, funded by the VKR/ Carlsberg foundations, he worked at Argonne National Laboratory near Chicago. He is now a senior scientist at Technical University of Denmark, working on methodologies for X-FEL experiments and new tomographic imaging modalities. 